\title{
Treatment of patients with severe traumatic brain injury: a 7-year single institution experience
}

Corrado P. Marini, MD, FACS ${ }^{\text {; }}$ Patrizio Petrone, MD, PhD, FACS ; John McNelis, MD, FACS, FCCM ${ }^{1}$; Erin Lewis, MD, MPH ${ }^{1}$; Anna Liveris, MD'; Michael F. Stiefel, MD, PhD ${ }^{3}$

'Department of Surgery, Jacobi Medical Center, Albert Einstein College of Medicine, Bronx, NY, USA

${ }^{2}$ Department of Surgery, NYU Langone Hospital-Long Island, NYU Long Island School of Medicine, Mineola, NY, USA

${ }^{3}$ Department of Neurosurgery, Piedmont Atlanta Hospital, Piedmont Healthcare, Atlanta, GA, USA

\section{ORIGINAL ARTICLE}

\section{Care}

Revised: March 09, 2021

Accepted: March 16, 2021

Corresponding Author:

Patrizio Petrone, MD, PhD, FACS

Department of Surgery, NYU Langone

Hospital-Long Island, NYU Long Island

School of Medicine, 222 Station Plaza

N., Suite 300, Mineola, NY 11501, USA

Tel: +1-516-663-9571

Fax: +1-516-663-8707

E-mail: patrizio.petrone@nyulangone.org

Background: This study was designed to compare the efficacy of multimodality monitoring and goal-directed therapy protocol (MM\&GDTP), in patients with Glasgow Coma Scale (GCS) scores $\leq 8$ with the conventional intracranial pressure (ICP)-cerebral perfusion pressure (CPP) treatment.

Methods: The study was divided into two time periods, a 2-year historic period in which severe traumatic brain injury (sTBI) patients were treated with an ICP-CPP targeted strategy and a 5-year intervention period during which MM\&GDTP was utilized. Patients with unsurvivable brain injuries were excluded. Variables of interest included mechanism of injury, age, sex, hemodynamics, GCS score, abbreviated injury score-head (AIS-H), Marshall Class, injury severity score, decompressive craniectomy, ventilator/intensive care unit days, length of stay, predicted mortality by corticosteroid randomization after significant head injury model, functional outcome, and mortality.

Results: The study group comprised 810 sTBI patients, aged 14-93 years, admitted during a 7-year period; of these patients, 67 and 99 AIS- $\mathrm{H} \geq 4$ and Marshall Class $\geq 111$ were included in control and intervention groups, respectively. The control group was treated with an ICP-CPP targeted approach, while the intervention group with an MM\&GDTP. At presentation and after resuscitation, patients in the intervention group required a higher CPP to reach the endpoints of therapy. The MM\&GDTP decreased mortality from $34.3 \%$ to $23.2 \%$, yielding a $32.3 \%$ improvement in overall survival and improved functional outcome as measured by Glasgow Outcome Scale $>3$ (MM\&GDTP vs. ICP-CPP: 50/99 vs. 15/67, P=0.003).

Conclusion: Institution of MM\&GDTP targeted to threshold-defined values improves functional outcomes and may reduce mortality among patients with sTBI compared to that of patients receiving an ICP-CPP-based treatment.

Keywords: Traumatic brain injuries; Severe; Treatment; Multimodality monitoring and goal-directed therapy protocol; Mortality 


\section{INTRODUCTION}

The treatment of patients with severe traumatic brain injury (sTBI) remains controversial. There is a wide range of perspectives on the value of foregoing intracranial pressure (ICP) monitoring and treating patients based solely on clinical and radiological findings versus utilizing either an intracranial pressure-cerebral perfusion pressure (ICP-CPP) targeted approach or a multimodality monitoring and brain tissue oxygen tension (ICP-PbtO ${ }_{2}$ ) targeted treatment aimed at optimizing cerebral blood flow and brain tissue oxygenation. Regardless of modality employed, studies have confirmed that short periods of brain hypoxia with CPP $<60 \mathrm{mmHg}$ are associated with decreased survival and compromised functional recovery at 6 and 12 months, respectively [1-3]. The Brain Oxygen Optimization in Severe TBI, Phase 2 (BOOST2) trial suggested a potential benefit of an ICP-PbtO targeted treatment over an ICP-CPP targeted approach as the latter can reduce the duration and depth of brain hypoxia; however, it did not document a survival advantage for patients [4]. Following these findings, the BOOST- 3 prospective randomized trial was initiated to further demonstrate whether an ICP-PbtO ${ }_{2}$ targeted treatment is superior to an ICP-CPP targeted approach from the standpoint of overall mortality and functional outcome; this study is ongoing [5]. At this time, there is no evidence to support the use of $\mathrm{ICP}_{-} \mathrm{PbtO} \mathrm{O}_{2}$ targeted treatment for all patients with sTBI.

Following the recruitment of two of the authors of this study (MFS and CPM), a multimodality monitoring and goal-directed therapy protocol (MM\&GDTP) was implemented in our Level I Trauma Center in 2011. The protocol includes a set of interventions aimed at preventing secondary brain injury and maintaining functional brain metabolism during the first 5 days following injury. It uses a tiered intervention strategy to treat isolated increases in ICP and decreased cerebral blood flow identified with near-infrared spectroscopy (NIRS) regional cerebral oxygen saturation $\left(\mathrm{rSO}_{2}\right)$ using cerebral microdialysis (CMD) data to detect brain tissue hypoxia. Our initial experience with MM\&GDTP showed an improved survival compared to that predicted by the corticosteroid randomization after significant head injury (CRASH) model, but we did not compare contemporary outcomes with those of historical controls from our own institution $[6,7]$. Therefore, this study compared the mortality and functional outcomes of patients with sTBI who were treated during a pre-MM\&GDTP control period of 2 years with a subsequent 5-year period, during which the MM\&GDTP was implemented.

\section{METHODS}

This study involved a retrospective chart review, and an informed consent waiver was granted. This study compared the outcomes of patients (age range, 14-93 years) with sTBI treated at a single institution over a period of 7 years. It was divided into two time periods: a historic control period that extended from January 1, 2009 to December 31, 2010 and an intervention period from April 1, 2011 to December 31, 2015, during which patients with sTBI were treated with a 5-day MM\&GDTP. The decision to limit the control group to those treated during these 2 years was based on the presence of a different group of neurosurgeons before that period. Patients excluded from the analysis included those treated between January 1, 2011 and March 31, 2011 because not all components of the MM\&GDTP were available until April 1, 2011. Patients with one or more of the following criteria, after resuscitation, physiological stabilization, and a period of observation who were deemed to have suffered an unsurvivable brain injury were excluded from the study: (1) Glasgow Coma Scale (GCS) score 3 with dilated and fixed pupils, (2) GCS score 3 with pupils unreactive to light, (3) GCS score 3 and brain injury observed on a computed tomography (CT) scan of the head expected to result in permanent loss of all brain function above the brain stem, and (4) brain perfusion CT scan showing absence of cerebral blood flow.

Multimodality monitoring included monitoring of ICP, CPP, which was defined as the difference between the mean systemic blood pressure and ICP, $\mathrm{PbtO}_{2}$ (Integra Licox Brain Tissue Monitoring; Integra LifeSciences, Princeton, NJ, USA) in mmHg; $\mathrm{rSO}_{2}$ by NIRS (Medtronic \& Covidien, Minneapolis, MN, USA); and hourly CMD data. The goal-directed therapy protocol included maintenance of normothermia $\left(37^{\circ} \mathrm{C}\right)$ with dry water immersion (Arctic Sun 5000 Temperature Management System, Medivance-Bard, Louisville, CO, USA), $\mathrm{PbtO}_{2} \geq 20 \mathrm{mmHg}$, ICP $\leq 20$ $\mathrm{mmHg}, \mathrm{CPP} \geq 70 \mathrm{mmHg}, \mathrm{rSO}_{2} \geq 55 \%$, CMD lactate/pyruvate ratio (LPR) $<40$, nutritional support targeted to ensure a respiratory quotient between 0.83 and 0.87 by indirect calorimetry on days 3 and 5, with calories adjustment as needed based on the day 3 respiratory quotient, and positive nitrogen balance by day 7 . To achieve the threshold CPP value required to keep $\mathrm{PbtO}_{2}$ and CMD LPR above the critical values, when needed, patients received a limited amount of normal saline (NS) $(1,000 \mathrm{~mL})$ and norepinephrine infusion at a dose of 0.1 to $0.2 \mu \mathrm{g} / \mathrm{kg} / \mathrm{min}$. Post-pyloric peptide-based enteral nutrition was initiated upon completion of the resuscitation phase. All patients were sedated to synchrony with the ventilator, avoidance of cough and a modified Ramsey score of 2 achieved with midazolam and propofol infu- 
sion. Patients were treated using a 2-tier approach based on their classification (Fig. 1). Osmotherapy included administration of $3 \%$ saline and the addition of either low- or high-dose mannitol when appropriate. Burst suppression, monitored with continuous electroencephalography, was initiated with an infusion of midazolam at a dose up to $15 \mathrm{mg} / \mathrm{hr}$ and propofol at a dose up to 100 $\mu \mathrm{g} / \mathrm{kg} / \mathrm{min}$ when ICP, low $\mathrm{PbtO}_{2}$, or low $\mathrm{rSO}_{2}$ were not responsive to first-tier therapy. The anti-shivering protocol used during the normothermic approach involved the use of a Bair Hugger set at $4^{\circ} \mathrm{C}$ with administration of a combination of acetaminophen, buspirone, fentanyl, midazolam, and propofol, and when needed, the addition of cisatracurium.

Indications for immediate craniotomy included symptoms of uncal herniation syndrome, midline shift $>1 \mathrm{~cm}$, epidural hema- toma volume $>30-40 \mathrm{~mL}$, presence of subdural hematoma with thickness $>0.5 \mathrm{~cm}$ extending over the hemispheres or $>30-40$ $\mathrm{mL}$ volume with a significant mass effect, and intraparenchymal hemorrhage $>30 \mathrm{~mL}$ in the temporal lobe or cerebellum with a significant mass effect. A large fronto-temporoparietal ( $\geq 12 \times 15$ $\mathrm{cm}$ ) decompressive craniectomy was employed for patients with late refractory ICP that did not respond to second-tier treatment modalities. All intracranial monitors were inserted within 2-4 hours of sTBI diagnosis, except for patients who went directly to the operating room for evacuation of mass lesions and had monitors placed after the craniotomy.

A multi-channel monitor (Moberg ICU Solutions, Ambler, PA, USA) provided continuous online recording of all multimodality monitoring minutes of brain flow and oxygen variables. $\mathrm{CMD}$ was

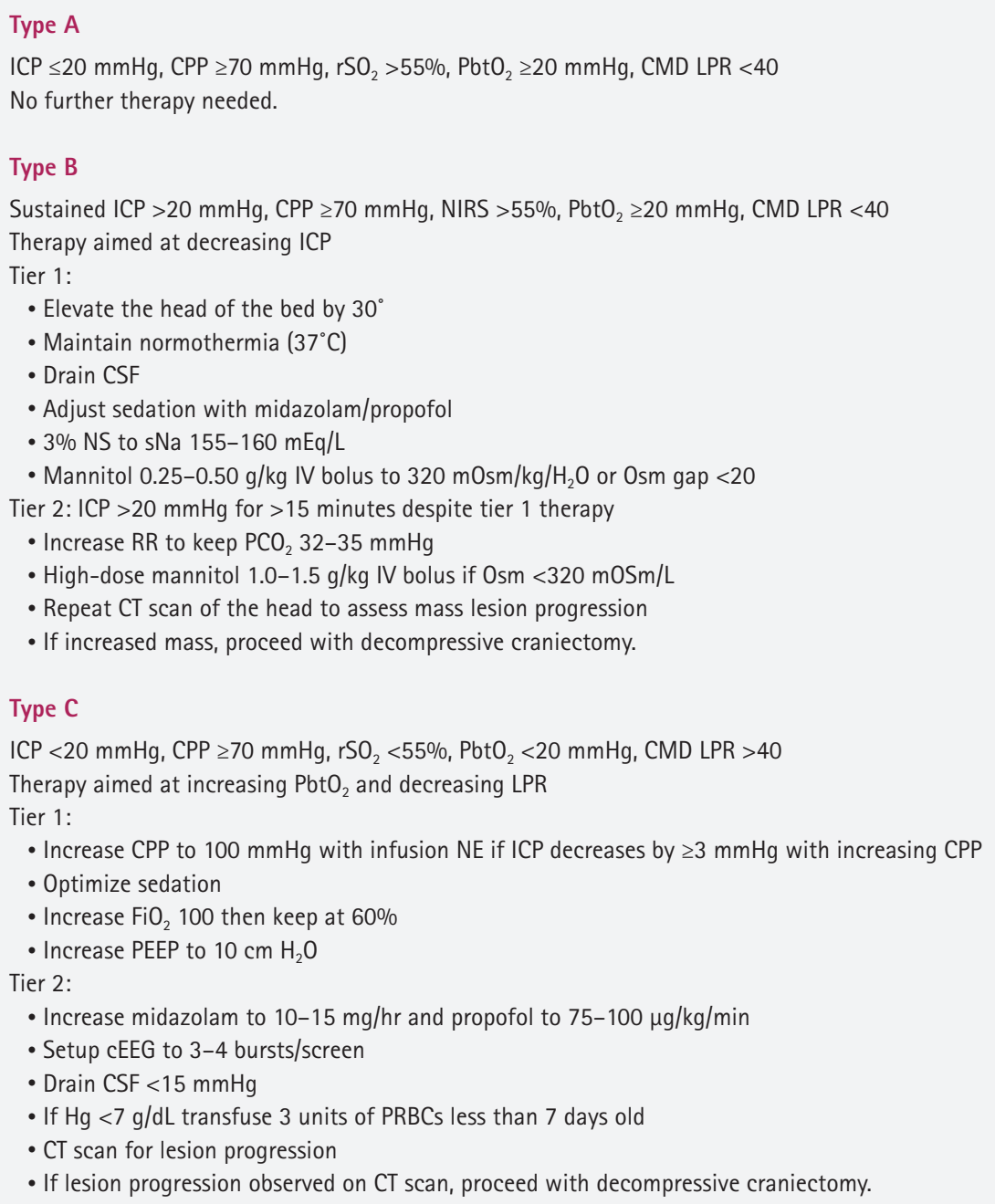

Fig. 1. Treatment algorithm. ICP, intracranial pressure; $\mathrm{CPP}$, cerebral perfusion pressure; $\mathrm{rSO}_{2}$, regional cerebral oxygen saturation; $\mathrm{PbtO}_{2}$ brain tissue oxygen tension; CMD, cerebral microdialysis; LPR, lactate/pyruvate ratio; NIRS, near-infrared spectroscopy; CSF, cerebrospinal fluid; NS, normal saline; sNa, seum sodium; IV, intravenous; RR, respiratory rate; $C T$, computed tomography; NE, norepinephrine; PEEP, positive end-expiratory pressure; cEEG, continuous electroencephalogram; Hg, hemoglobin; PRBC, packed red blood cell. 
performed via a dual lumen catheter inserted concurrently with the Licox catheter. CMD data that were measured included levels of glucose (normal value, $14.4-46.8 \mathrm{mmol} / \mathrm{L})$, lactate $(2.0-3.8$ $\mathrm{mmol} / \mathrm{L})$, pyruvic acid $(119-213 \mu \mathrm{mol} / \mathrm{L})$, glutamate $(0.0-32.0$ $\mu \mathrm{mol} / \mathrm{L})$, and glycerol (38.0-126 $\mu \mathrm{mol} / \mathrm{L})$ and LPR. CMD data and mean values of the measurements were averaged hourly. All patients with a GCS score $<10$ on day 5 underwent tracheostomy with placement of a subglottic suctioning tracheostomy tube and percutaneous endoscopic gastrostomy between days 5 and 7 .

Treatment during the control period included maintenance of CPP $\geq 60 \mathrm{mmHg}$ and ICP monitoring with external ventricular drainage, control of increased ICP with drainage of CSF, infusion of $3 \% \mathrm{NS}$ titrated to a serum sodium concentration of $155 \mathrm{mEq} / \mathrm{L}$, and the infusion of mannitol titrated to $320 \mathrm{mOsm} / \mathrm{L}$ or an osmolar gap $<20$ as rescue therapy for increased ICP. The timing and performance of the decompressive craniectomy were at the discretion of the individual neurosurgeon. $\mathrm{PbtO}_{2}$ monitoring and CMD were not performed, and normothermia was not protocolized. Hyperthermia was controlled on an individual basis, and enteral nutrition was administered at the discretion of the individual neurosurgeon. Indirect calorimetry and nitrogen balance measurements were not monitored. The timings of tracheostomy and percutaneous endoscopic gastrostomy were decided by the individual neurosurgeon.

Data acquired included mechanism of injury; age; sex; hemodynamics; GCS scores on admission and upon completion of the resuscitation phase and a period of physiological stabilization and observation; abbreviated injury score-head (AIS-H); Marshall Class; injury severity score (ISS); performance of decompressive craniectomy; ventilator and intensive care unit days; length of stay; predicted mortality (PM) by the CRASH model; and actual mortality. The GCS score obtained after completion of the resuscitation phase and a period of physiological stabilization and observation was used for statistical analysis because of its improved prognostic value over the GCS score acquired on admission. Continuous data are presented as means with standard deviation, and nonparametric data are presented as medians with interquartile range (IQR). Statistical analysis was limited to the comparison of the 99 patients who underwent treatment with the MM\&GDTP with a control group of 67 patients who underwent conventional ICP-CPP treatment. Of note, all but one (MFS) of the neurosurgeons treating the patients during the two study intervals remained the same; however, during the intervention period, the neurosurgeons agreed to treat the patients with the MM\&GDTP. Analysis of continuous data was performed with an unpaired $t$-test, and analysis of categorical data was performed with a chisquare or Fisher's exact test. Medians were compared using a non- parametric Mann-Whitney $U$-test. Stepwise logistic regression analysis was performed to identify variables predictive of outcome. The inverse probability of treatment weighted adjusted logistic regression models (SAS ver. 9.4; SAS Institute, Cary, NC, USA) were used to study the effect of MM\&GDTP on mortality and functional outcomes. Statistical significance was accepted to correspond to a $P$-value less than 0.05 .

\section{RESULTS}

A total of 810 patients with sTBI were admitted to our Level I Trauma Center between January 1, 2009 and December 31, 2015. One hundred and twenty-three patients died between days 1 and 2 or progressed to brain death from unsurvivable brain injury, and 316 patients with AIS-H < 4 were excluded; therefore, 371 patients were included for further analysis. An additional $205 \mathrm{pa}-$ tients with AIS $>0$ in other body regions were excluded to limit the analysis to patients with isolated sTBI. Of the 166 remaining patients, 67 (control group) were treated with an ICP-CPP targeted approach following the Brain Trauma Foundation guidelines, whereas the 99 patients in the intervention group were treated with the MM\&GDTP (Fig. 2); the patients were matched for sex,

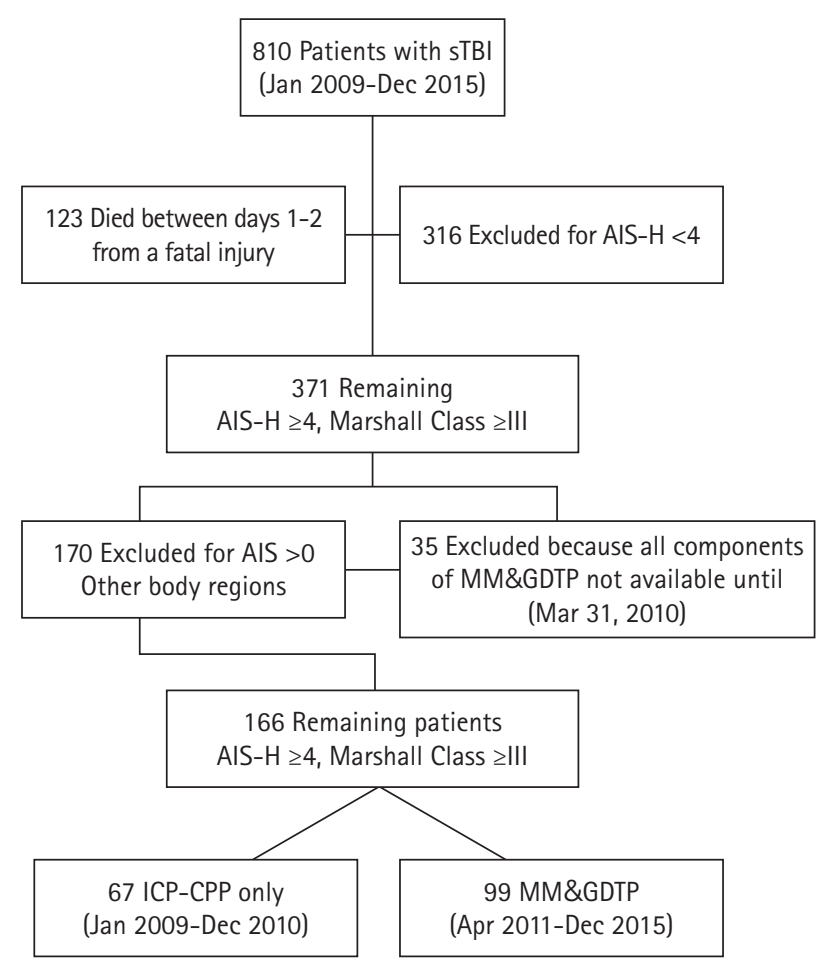

Fig. 2. Study design flowchart. sTBI, severe traumatic brain injury; AIS-H, abbreviated injury score-head; MM\&GDTP, multimodality monitoring and goal-directed therapy protocol; ICP-CPP only, intracranial pressure-cerebral perfusion pressure targeted approach. 
Table 1. Patients characteristics

\begin{tabular}{|c|c|c|c|c|}
\hline Variable & All patients $(n=166)$ & MM\&GDTP $(n=99)$ & ICP-CPP only $(n=67)$ & $P$-value \\
\hline Age (yr) & $56 \pm 23$ & $59 \pm 22$ & $51 \pm 23$ & 0.02 \\
\hline Male:female & $121: 45(72.9 / 27.1)$ & $70: 29(70.7 / 29.3)$ & $51: 16(76.1 / 23.9)$ & 0.48 \\
\hline $\mathrm{SBP}(\mathrm{mmHg})$ & $143 \pm 30$ & $146 \pm 31$ & $138 \pm 28$ & 0.08 \\
\hline GCS score & $3(3-5)$ & $3(3-3)$ & $3(3-6)$ & 1 \\
\hline AIS-H 4 & 84 & $53(53.5)$ & $31(42.3)$ & 0.42 \\
\hline AIS-H 5 & 82 & $46(46.5)$ & $36(57.7)$ & 0.42 \\
\hline DC & $35(21.0)$ & $21(21.2)$ & $14(20.9)$ & 1 \\
\hline Marshall Class III & 53 (31.9) & $32(32.3)$ & $21(31.3)$ & 1 \\
\hline Marshall Class IV & $34(20.4)$ & $20(20.2)$ & $14(20.9)$ & 1 \\
\hline Marshall Class V & $35(21.0)$ & $21(21.2)$ & $14(20.9)$ & 1 \\
\hline Marshall Class VI & $44(26.7)$ & $26(26.3)$ & $18(26.9)$ & 1 \\
\hline GOS score & $3(1-4)$ & $4(2-4)$ & $2(1-3)$ & 0.01 \\
\hline GOS score $>3$ & 65 (39.2) & $50(50.5)$ & $15(22.4)$ & 0.003 \\
\hline
\end{tabular}

Values are presented as mean \pm standard deviation, number (\%), or median (interquartile range).

MM\&GDTP, multimodality monitoring and goal-directed therapy protocol; ICP, intracranial pressure; CPP, cerebral perfusion pressure; SBP, systolic blood pressure; GCS, Glasgow Coma Scale; AIS-H, abbreviated injury score-head; ISS, injury severity score; DC, decompressive craniectomy; PM, predicted mortality; CRASH, corticosteroid randomization after significant head injury; GOS, Glasgow Outcome Scale.

Table 2. Covariates by weight

\begin{tabular}{|c|c|c|c|c|c|c|}
\hline \multirow{2}{*}{ Characteristics } & \multicolumn{3}{|c|}{ Unweighted } & \multicolumn{3}{|c|}{ Weighted by inverse probability of treatment } \\
\hline & ICP-CPP & MM\&GDTP & SMD & ICP-CPP & MM\&GDTP & SMD \\
\hline Age & $59 \pm 22$ & $51 \pm 23$ & -0.367 & $57 \pm 37$ & $56 \pm 30$ & 0.063 \\
\hline ED SBP $(\mathrm{mmHg})$ & $138 \pm 28$ & $146 \pm 31$ & -0.275 & $143 \pm 45$ & $143 \pm 40$ & -0.003 \\
\hline ED GCS & $3.7 \pm 1.5$ & $4.4 \pm 1.9$ & -0.417 & $4.1 \pm 3.0$ & $4.1 \pm 2.3$ & 0.023 \\
\hline Sex & & & 0.023 & & & -0.043 \\
\hline Male & $51(76.1)$ & $70(70.7)$ & & $125(74.6)$ & $120(72.6)$ & \\
\hline Female & $16(23.9)$ & $29(29.3)$ & & $43(25.4)$ & $45(27.4)$ & \\
\hline AIS-H & & & -0.166 & & & -0.043 \\
\hline 4 & $31(46.3)$ & $53(53.5)$ & & $82(48.4)$ & $84(50.5)$ & \\
\hline 5 & $36(53.7)$ & $46(46.5)$ & & $87(51.6)$ & $82(49.5)$ & \\
\hline Decompressive craniectomy & & & 0.008 & & & -0.003 \\
\hline 0 & $53(79.1)$ & $78(78.8)$ & & $131(77.8)$ & $129(77.9)$ & \\
\hline 1 & 14 (20.9) & $21(21.2)$ & & $37(22.2)$ & $36(22.1)$ & \\
\hline Logit of the propensity score & & & 0.650 & & & -0.024 \\
\hline
\end{tabular}

Values are presented as mean \pm standard deviation or number (\%). SMD were used to assess the balance of the covariates before and after inverse probability of treatment weighting between the ICP-CPP and MM\&GDTP groups. An SMD value $<0.1$ was accepted to correspond to adequate balance. ICP, intracranial pressure; CPP, cerebral perfusion pressure; MM\&GDTP, multimodality monitoring and goal-directed therapy protocol; SMD, standardized mean difference; ED, emergency department; SBP, systolic blood pressure; GCS, Glasgow Coma Scale; AIS-H, abbreviated injury score-head.

GCS score, ISS, AIS-H, Marshall Class, decompressive craniectomy, and PM by the CRASH model.

The pre- and post-propensity matching variables for the two groups are shown in Tables 1 and 2. Before propensity matching, patients in the intervention group were significantly older than those in the control group. As shown in Table 1, there was an al- most equal distribution of patients with Marshall Class III to VI between the two groups. The patients in the intervention group required a higher CPP of $83 \pm 6 \mathrm{mmHg}$ to achieve a $\mathrm{rSO}_{2}>55 \%$ with $\mathrm{PbtO}_{2} \geq 20 \mathrm{mmHg}$ and a CMD LPR $<40$ with pyruvate $<120 \mathrm{mmol} / \mathrm{L}$ and glucose $<8 \mathrm{mmol} / \mathrm{L}$, compared to patients in the control group, which required a mean of $66 \pm 4 \mathrm{mmHg}$ to stay 
within the target CPP of $60-70 \mathrm{mmHg}$ and ICP $\leq 20 \mathrm{mmHg}$. The percentage of patients who required decompressive craniectomy did not differ between the two study periods. As seen in Table 3, there were no differences in age, sex distribution, GCS score, ISS, and PM between the patients in the two groups undergoing decompressive craniectomy, but more patients in the intervention group had an AIS-H 5 compared to that in the control group. While there was a lower mortality in the patients who underwent decompressive craniectomy during the MM\&GDTP period, the difference did not achieve statistical significance.

The patients in the intervention group had a lower mortality rate than that of patients in the control group (23/99 [23.2\%] vs. 23/67 [34.3\%], respectively). However, the difference was not statistically significant. Nevertheless, there was a $32.4 \%$ reduction in mortality when patients treated during the two intervals were compared. Time to death was not significantly different between the two periods, 10 days (IQR, 7-16 days) as opposed to 8 days (IQR, 6-14 days), control versus intervention period, respectively $(P>0.05)$. The analysis of mortality, stratified by Marshall Class, suggested a statistically significant improvement in mortali- ty in patients with Marshall Class V pathology (Table 4). The mortality of patients with sTBI during the intervention interval was ascribable to sustained severe metabolic crisis defined by an LPR $>40$ in patients with a pyruvate level $<120 \mu \mathrm{mol} / \mathrm{L}$ and a glucose level $<8 \mathrm{mmol} / \mathrm{L}$ in the CMD effluent, despite the achievement of target levels for brain flow variables in some of the patients.

\section{Functional outcome}

Patients treated with the MM\&GDTP had a statistically significant improvement in functional outcome (Glasgow Outcome Scale $[$ GOS] score $>3$ ) at the time of transfer to different TBI rehabilitation centers compared to patients treated with the ICPCPP targeted approach. Fifty of 99 patients (50.5\%) treated as per the MM\&GDTP, as opposed to 15 of 67 (22.4\%) patients treated with the ICP-CPP targeted approach, had a GOS score $>3$ at a median of 18 days (IQR, 12-27 days) and 23 days (IQR, 16-36 days) $(P<0.05)$. As seen in Table 5 , the improved functional outcome was observed mostly in the group of patients treated with the MM\&GDTP who did not undergo decompressive craniecto-

Table 3. Characteristics of patients who underwent decompressive craniectomy

\begin{tabular}{|c|c|c|c|}
\hline Variable & Historic control group $(n=14 / 67,20.9 \%)$ & MM\&GDTP group $(n=21 / 99,21.2 \%)$ & $P$-value \\
\hline Age (yr) & $48 \pm 14$ & $51 \pm 22$ & 0.65 \\
\hline Male sex & 113 & 156 & 0.71 \\
\hline GCS score & $3(3-3)$ & $3(3-3)$ & 1 \\
\hline ISS & $22 \pm 4$ & $22 \pm 4$ & 1 \\
\hline AIS-H 4:5 & $5: 9(35.7: 64.3)$ & $6: 15(28.6: 71.4)$ & 0.22 \\
\hline Vent-day & $10(6-21)$ & $8(4-16)$ & 1 \\
\hline LOS (day) & $19(8-31)$ & $17(10-24)$ & 0.45 \\
\hline PM (\%) & $59 \pm 21$ & $55 \pm 25$ & 0.62 \\
\hline Mortality & $7(50.0)$ & $9(42.8)$ & 0.73 \\
\hline
\end{tabular}

Values are presented as mean \pm standard deviation, median (interquartile range), or number (\%).

MM\&GDTP, multimodality monitoring and goal-directed therapy protocol; SBP, systolic blood pressure; GCS, Glasgow Coma Scale; ISS, injury severity score; AIS-H, abbreviated injury score-head; GOS, Glasgow Outcome Scale; Vent-day, number of ventilator days; LOS, length of hospital stay; PM, predicted mortality.

Table 4. Mortality stratified by Marshall Class

\begin{tabular}{lcccr}
\hline Marshall Class & Number & Overall mortality & ICP-CPP alone mortality & MM\&GDTP mortality \\
\hline III & $53(31.9)$ & $7 / 53(13.2)$ & $1 / 21(4.7)$ & $6 / 32(18.7)$ \\
IV & $34(20.5)$ & $7 / 34(20.2)$ & $4 / 14(28.6)$ & $3 / 20(15.0)$ \\
V & $35(21.1)$ & $10 / 35(28.6)$ & $8 / 14(57.1)$ & $2 / 21(9.5)^{a)}$ \\
VI & $44(26.5)$ & $22 / 44(50.0)$ & $10 / 18(55.5)$ & $12 / 26(46.1)$ \\
Total & 166 & $46 / 166(27.7)$ & $23 / 67(34.3)$ & $23 / 99(23.2)$ \\
\hline
\end{tabular}

Values are presented as number (\%).

ICP, intracranial pressure; CPP, cerebral perfusion pressure; MM\&GDTP, multimodality monitoring and goal-directed therapy protocol.

a) $P<0.05$. 
Table 5. Functional outcome stratified by decompressive craniectomy status

\begin{tabular}{lccc}
\hline \multirow{2}{*}{ Group } & \multicolumn{3}{c}{ GOS score $>3$} \\
\cline { 2 - 4 } & $\begin{array}{c}\text { No decompressive } \\
\text { craniectomy }\end{array}$ & $\begin{array}{c}\text { Decompressive } \\
\text { craniectomy }\end{array}$ & Total \\
\hline ICP-CPP & $11 / 53(20.7)$ & $4 / 14(28.6)$ & $15 / 67(22.4)$ \\
MM\&GDTP & $45 / 78(57.7)$ & $5 / 21(23.8)$ & $50 / 99(50.5)$ \\
\hline
\end{tabular}

Values are presented as number (\%).

GOS, Glasgow Outcome Scale; ICP, intracranial pressure; CPP, cerebral perfusion pressure; MM\&GDTP, multimodality monitoring and goaldirected therapy protocol.

my. In contrast, patients in the ICP-CPP group showed similar functional outcomes, independent of the performance of decompressive craniectomy. Of note, AIS-H and age were the only two variables predictive of functional outcome and mortality with the following odds ratios (OR) with $95 \%$ confidence intervals (CI): $5.12(2.80-9.33)$ and $1.02(1.01-1.04)$, respectively $(P<0.001)$. The inverse probability of treatment weighted logistic regression models with the following covariates: age (in years), systolic blood pressure, sex, GCS score (3-15), AIS-H (4-5), and decompressive craniectomy for the two dependent outcome variables, death and functional outcome (GOS score $>3$ ), showed that the MM\&GDTP did not improve mortality (OR, 0.66; 95\% CI, $0.40-1.07 ; P=0.089)$ but improved functional outcome by 3.5 fold (OR, 3.56; 95\% CI, 2.22-5.70; $P<0.001)$ compared to the ICP-CPP treatment strategy.

\section{DISCUSSION}

The treatment of patients with sTBI remains controversial from the standpoint of the best monitoring modalities and approach to reach specific endpoints of targeted therapy. This is a result of the conflicting conclusions of clinical trials, some of which showed that for patients with sTBI care based on clinical monitoring and imaging alone is as effective as maintaining monitored ICP $\leq 20$ $\mathrm{mmHg}$. However, other studies have showed a potential benefit for an ICP-PbtO ${ }_{2}$ targeted treatment over an ICP-CPP targeted strategy from the standpoint of reducing duration and depth of brain hypoxia but without a survival advantage. Moreover, some studies have shown a survival advantage at 6 months from decompressive craniectomy in patients with refractory intracranial hypertension but a higher rate of vegetative state, lower and upper severe disability than that seen with medical care; nevertheless, these have not shown any difference in the rates of moderate disability and good recovery between patients treated with decompressive craniectomy and those treated with medical therapy alone $[4,8,9]$.
Our approach for the treatment of patients with sTBI has evolved over time from an initial ICP-PbtO ${ }_{2}$ targeted therapy to one that includes maintenance of normothermia, early achievement of adequate nutritional support targeted to a positive nitrogen balance by day 7 using indirect calorimetry, in addition to reaching specific endpoints of brain flow and cerebral metabolism assessed using $\mathrm{PbtO}_{2}, \mathrm{rSO}_{2}$, and $\mathrm{CMD}$ data.

We have previously studied the effectiveness of the MM\&GDTP for the treatment of patients with sTBI by comparing our mortality rate to that predicted by the CRASH model [6]. We believe that this approach for analyzing our results led us to prematurely conclude about the superiority of our approach over the more conservative ones owing to the overestimation of mortality rate by the CRASH model and failure to compare our cohort to a historic control group of patients [7]. For this reason, we decided to assess the efficacy of our recent MM\&GDTP by comparing patients treated with this protocol to a historical group of patients treated in the same institution by the same neurosurgeons but without the new protocol. We limited our comparison to patients with the most severe form of isolated TBI, namely, AIS-H $\geq 4$ and Marshall Class $\geq$ III. While we still used the CRASH model to compare the mortality rate to the predicted one, we were interested in assessing whether the introduction of the MM\&GDTP would improve the mortality and functional outcome of patients with sTBI compared to those of a recent historical group of patients matched for the severity of TBI and treated by the same group of neurosurgeons with excellent results.

The implementation of the MM\&GDTP decreased the mortality rate among patients with sTBI, from $34.3 \%$ to $23.2 \%$, yielding a $32.3 \%$ reduction. However, the difference in mortality was not statistically significant. The treatment of patients using the MM\&GDTP provided a statistically significant benefit in functional outcome, as measured by the GOS. This improvement in functional outcome was observed mostly in the group of patients treated with the MM\&GDTP who did not undergo decompressive craniectomy. Patients treated with the MM\&GDTP who underwent decompressive craniectomy did not have improved functional outcome when compared with their counterparts in the ICP-CPP treated group. We believe that the failure of decompressive craniectomy to offer a benefit in functional outcome in both groups can be attributed to several reasons, such as the assessment of functional outcome at discharge from the hospital and at 6 months after discharge may be a premature endpoint for patients with sTBI. The outcomes of decompression continue to improve beyond 6 months, and possibly even beyond 12 months. Additionally, while decompressive craniectomy decreases ICP and improves brain compliance and cerebral blood flow, it may not pro- 
vide a benefit to patients with devastating structural brain lesions, such as those in the brainstem that may have not been detected on $\mathrm{CT}$ and instead could have been identified before decompression by magnetic resonance imaging. Another important reason why patients in the intervention group who underwent decompressive craniectomy did not have an improved mortality rate and favorable functional outcome could be because the secondary decompressive craniectomy was performed for "early" refractory ICP elevation, defined as an increased ICP $>20 \mathrm{mmHg}$ for $>15 \mathrm{~min}-$ utes not responsive to tier 2 therapy (Fig. 1). Early secondary decompressive craniectomy is no longer recommended for improving mortality and favorable outcomes [10].

Since there was no difference in the severity of the brain injury as per GCS score, AIS-H, and Marshall Class between the patients during the two intervals, we that the outcomes experienced by patients during the control period were due to the limitations of the ICP-CPP targeted approach, particularly the inability to identify a suboptimal $\mathrm{PbtO}_{2}$ leading to cerebral metabolic crisis despite the achievement of adequate ICP and CPP. In 2005, Stiefel et al. [11] demonstrated that the use of both ICP and brain tissue $\mathrm{PO}_{2}$ monitors and therapy directed at brain tissue $\mathrm{PaO}_{2}$ were associated with reduced patient death following sTBI. Subsequently, in 2006, the same investigators, in a study of 25 patients undergoing neurocritical care monitoring, demonstrated that $47 \%$ of patients had a $\mathrm{PbtO}_{2}<20 \mathrm{mmHg}$ despite a CPP and an ICP $\geq 60$ and $<25$ $\mathrm{mmHg}$, respectively, highlighting the limitations of the ICP-CPP approach for the treatment of patients with sTBI [12]. In 2010, Spiotta et al. [13] corroborated the beneficial impact of $\mathrm{Pb}$ $\mathrm{tO}_{2}$-guided therapy on the outcome of patients with sTBI. They reported a decrease in mortality from $45 \%$ in 53 patients whose therapy was guided by the ICP-CPP to $25 \%$ in 70 patients whose therapy was guided by $\mathrm{CPP}-\mathrm{PbtO}_{2}$.

We hypothesize that the poor functional outcomes of patients treated before the implementation of the MM\&GDTP is likely the result of the suboptimal information provided by ICP, as opposed to the additional information available from the $\mathrm{PbtO}_{2}$ and $\mathrm{CMD}$ data during the intervention period. Another reason for the poor outcomes during the control period may be related to the maintenance of CPP to values between 60 and $70 \mathrm{mmHg}$, which, based on the data provided by CMD, is often associated with metabolic crises and may lead to adverse outcomes, if sustained. The additional information available from the $\mathrm{PbtO}_{2}$ and $\mathrm{CMD}$ data can be used to optimize the individual level of CPP and, therefore, may limit the number of metabolic crisis events, which in turn may yield better outcomes.

The improved mortality experienced in the setting of a Level I trauma center already performing at an observed to expected ratio less than one for patients with sTBI may be attributed to the implementation of this MM\&GDTP since this was the only difference between the treatments of patients with sTBI in the two study intervals. It appears that the addition of more advanced monitoring methods, which can identify cerebral blood flow and brain oxygen crisis events, added to the armamentarium of a group of very competent neurosurgeons and surgical intensivists, provides a more sensitive methodology to monitor and minimize the number and duration of cerebral metabolic crises. This may in turn improve 30-day survival for patients with sTBI and, more importantly, their functional outcome.

ICP may represent a non-specific marker that highlights the dynamic changes occurring in the brain, and both waveform data and cerebrovascular reactivity index values can be derived from ICP monitoring, which may help guide therapy. However, a therapeutic strategy that uses ICP monitoring and CPP as endpoint of therapy may be less sensitive to the identification of brain oxygen crises related to brain flow and cerebral metabolism when compared to $\mathrm{PbtO}_{2}$ monitoring and the metabolic data provided by $\mathrm{CMD}$ to the identification of brain oxygen crises related to brain flow and cerebral metabolism [14].

As noted in our results, patients treated with the MM\&GDTP required a much higher CPP to provide the cerebral blood flow needed to avoid the development of cerebral metabolic crisis compared with the CPP of the patients treated with the ICP-CPP targeted therapy. However, despite the higher CPP, some of the patients who died during the intervention interval suffered several episodes of severe metabolic crisis, which were ultimately responsible for their death. As previously reported, severe metabolic crisis can occur even in the presence of adequate $\mathrm{CPP}, \mathrm{rSO}_{2}, \mathrm{PbtO}_{2}$, and ICP, although the number of these events and their duration is significantly lower in survivors than in non-survivors, who typically experience on average more than 2 hours of severe metabolic crisis when CPP and $\mathrm{PbtO}_{2}$ are less than 60 and $20 \mathrm{mmHg}$, respectively [7].

Some authors have reported an increased incidence of acute respiratory distress syndrome (ARDS) in patients with sTBI treated with hypervolemia to increase CPP $[15,16]$. Our patients did not experience any episode of ARDS despite our approach targeting $\mathrm{CPP}$ values much higher than previously reported. The method used to increase CPP may have played a role in the difference in the incidence of ARDS. The use of norepinephrine instead of large amounts of crystalloids to raise CPP may explain the difference between our results and those reported by other authors. It is likely that in the setting of a capillary leak syndrome triggered by trauma, as it occurs in STBI, the infusion of a large volume of crystalloids to raise CPP may lead to a higher incidence of ARDS as 
opposed to the incidence seen with an alternate approach that uses a vasoconstrictive agent to achieve a higher $\mathrm{CPP}$ to maintain $\mathrm{PbtO}_{2} \geq 20 \mathrm{mmHg}$ and $\mathrm{CMD} \mathrm{LPR}<40 \mathrm{mmHg}$.

Based on our institutional experience, we believe that the "optimal" CPP threshold is higher than the recommended 60-70 mmHg in patients with a $\mathrm{PbtO}_{2}<20 \mathrm{mmHg}$ and $\mathrm{rSO}_{2}<55 \%$. Additionally, the ICP response to increased CPP in patients with intact autoregulation is characterized by a significant decrease in ICP; therefore, attempts should be made for each individual patient with intact autoregulation to identify the "optimal" CPP. Targeting the treatment of patients with sTBI to this individualized "optimal" CPP may be more effective than the treatments targeting existing consensus-based guideline thresholds. Two prospective pilot studies evaluating CPP-optimized tailored therapies in different settings have demonstrated an improvement in patient physiology and outcomes $[17,18]$.

Our study has many limitations that prevent us from suggesting that our conclusions are generalizable. First, it represents a single institution's 7-year experience, with the control group being limited to those treated only during 2 years, and it is possible that the results are affected by the difference in the number of patients between the two study periods. The second limitation is the inability to identify which of the several threshold target values included in the MM\&GDTP may have affected the improvement in mortality and functional outcome, and whether the observed improvements were due to a Hawthorne effect during the intervention period of the study. Third, the inherent increased complexity of the MM\&GDTP compared to the ICP-CPP targeted treatment may have led to a much more intense overall monitoring and treatment and, possibly, fewer episodes of hypoperfusion during the intervention period compared to that during the historic control period. The fourth limitation is the absence of 6- and 12-month data regarding the overall functional outcome of the surviving patients in both groups.

The results of our study suggest that a therapeutic strategy based on a MM\&GDTP targeting threshold-defined values may reduce the mortality and improve the functional outcome of patients with sTBI, compared to those seen with an ICP-CPP treatment strategy, by enhancing cerebral oxygenation and brain metabolism through the optimization of cerebral blood flow. However, we believe that much larger studies are needed to understand the role of the MM\&GDTP as a whole and of each individual component to reveal the patient-specific brain injury pattern in order to formulate an individual optimal treatment plan. Monitoring devices themselves will not improve outcomes; they are needed to identify specific physiological patterns of survivorship and improved functional outcomes that can be used for the timely im- plementation of individualized treatment for patients with sTBI.

\section{ARTICLE INFORMATION}

\section{Ethics statement}

The New York Medical College Institutional Review Board approved the study design and methodology (L-11,356). This study is a retrospective chart review, and as such, there is no need for an informed consent.

\section{Conflict of interest}

No potential conflict of interest relevant to this article.

\section{ORCID}

Patrizio Petrone https://orcid.org/0000-0002-7629-1663

\section{Author contributions}

Conceptualization: CPM, MFS. Data curation: CPM. Formal analysis: CPM. Methodology \& Project administration: CPM, PP. Visualization: CPM, MFS. Writing-original draft: CPM, PP. Writingreview \& editing: CPM, PP, JMcN, EL, AL, MFS.

\section{REFERENCES}

1. Chesnut RM, Marshall LF, Klauber MR, Blunt BA, Baldwin N, Eisenberg HM, et al. The role of secondary brain injury in determining outcome from severe head injury.J Trauma 1993;34: 216-22.

2. Spaite DW, Hu C, Bobrow BJ, Chikani V, Sherrill D, Barnhart B, et al. Mortality and prehospital blood pressure in patients with major traumatic brain injury: implications for the hypotension threshold. JAMA Surg 2017;152:360-8.

3. Citerio G, Stocchetti N, Cormio M, Beretta L. Neuro-Link, a computer-assisted database for head injury in intensive care. Acta Neurochir (Wien) 2000;142:769-76.

4. Okonkwo DO, Shutter LA, Moore C, Temkin NR, Puccio AM, Madden CJ, et al. Brain oxygen optimization in severe traumatic brain injury phase-II: a phase II randomized trial. Crit Care Med 2017;45:1907-14.

5. Barsan W. Brain oxygen optimization in severe TBI, phase 3 (BOOST 3). ClinicalTrials.gov Identifier: NCT03754114. Bethesda: U.S. National Library of Medicine; 2021.

6. MRC CRASH Trial Collaborators, Perel P, Arango M, Clayton T, Edwards P, Komolafe E, et al. Predicting outcome after traumatic brain injury: practical prognostic models based on large cohort of international patients. BMJ 2008;336:425-9.

7. Marini CP, Stoller C, Shah O, Policastro A, Lombardo G, Asen- 
sio JA, et al. The impact of early flow and brain oxygen crisis on the outcome of patients with severe traumatic brain injury. Am J Surg 2014;208:1071-7.

8. Chesnut RM, Temkin N, Carney N, Dikmen S, Rondina C, Videtta W, et al. A trial of intracranial-pressure monitoring in traumatic brain injury. N Engl J Med 2012;367:2471-81.

9. Hutchinson PJ, Kolias AG, Timofeev IS, Corteen EA, Czosnyka M, Timothy J, et al. Trial of decompressive craniectomy for traumatic intracranial hypertension. N Engl J Med 2016;375: 1119-30.

10. Hawryluk GW, Rubiano AM, Totten AM, O'Reilly C, Ullman JS, Bratton SL, et al. Guidelines for the management of severe traumatic brain injury: 2020 update of the decompressive craniectomy recommendations. Neurosurgery 2020;87:427-34.

11. Stiefel MF, Spiotta A, Gracias VH, Garuffe AM, Guillamondegui $\mathrm{O}$, Maloney-Wilensky $\mathrm{E}$, et al. Reduced mortality rate in patients with severe traumatic brain injury treated with brain tissue oxygen monitoring. J Neurosurg 2005;103:805-11.

12. Stiefel MF, Udoetuk JD, Spiotta AM, Gracias VH, Goldberg A, Maloney-Wilensky E, et al. Conventional neurocritical care and cerebral oxygenation after traumatic brain injury. J Neurosurg 2006; 105:568-75.

13. Spiotta AM, Stiefel MF, Gracias VH, Garuffe AM, Kofke WA, Maloney-Wilensky E, et al. Brain tissue oxygen-directed man- agement and outcome in patients with severe traumatic brain injury. J Neurosurg 2010;113:571-80.

14. Le Roux P, Menon DK, Citerio G, Vespa P, Bader MK, Brophy GM, et al. Consensus summary statement of the international multidisciplinary consensus conference on multimodality monitoring in neurocritical care: a statement for healthcare professionals from the Neurocritical Care Society and the European Society of Intensive Care Medicine. Neurocrit Care 2014;21 Suppl 2:S1-26.

15. Rosner MJ, Rosner SD, Johnson AH. Cerebral perfusion pressure: management protocol and clinical results. J Neurosurg 1995;83:949-62.

16. Hendrickson CM, Howard BM, Kornblith LZ, Conroy AS, Nelson MF, Zhuo $\mathrm{H}$, et al. The acute respiratory distress syndrome following isolated severe traumatic brain injury. J Trauma Acute Care Surg 2016;80:989-97.

17. Jaeger M, Dengl M, Meixensberger J, Schuhmann MU. Effects of cerebrovascular pressure reactivity-guided optimization of cerebral perfusion pressure on brain tissue oxygenation after traumatic brain injury. Crit Care Med 2010;38:1343-7.

18. Dias C, Silva MJ, Pereira E, Monteiro E, Maia I, Barbosa S, et al. Optimal cerebral perfusion pressure management at bedside: a single-center pilot study. Neurocrit Care 2015;23:92-102. 